EPJ Web of Conferences 17, 07003 (2011)

DOI: $10.1051 /$ epjconf/20111707003

(C) Owned by the authors, published by EDP Sciences, 2011

\title{
Studies of light neutron-excess systems from bounds to continuum
}

\author{
Makoto Ito, \\ Department of Pure and Applied Physics, Kansai University, Yamate-cho, Suita, Japan, 564-8680
}

\begin{abstract}
The generalized two-center cluster model (GTCM), which can handle various single particle config urations in general two center systems, is applied to the light neutron-rich system, ${ }^{12} \mathrm{Be}=\alpha+\alpha+4 N$. We discuss the change of the neutrons' configuratio around two $\alpha$-cores as a variation of an excitation energy. We show that the covalent, ionic and atomic configuration appear in the unbound region above the $\alpha+{ }^{8} \mathrm{He}_{\text {g.s. }}$ particledecay threshold. The GTCM calculation is also applied to even Be isotopes, and the systematics on the structural changes from bound region to continuum is discussed.
\end{abstract}

\section{Introduction}

In the last two decades, developments of experiments with secondary RI beam have extensively advanced the studies on light neutron-rich nuclei. In particular, much efforts have been devoted to the investigation of molecular structure in $\mathrm{Be}$ isotopes. The $\mathrm{Be}$ isotopes can be considered as typical examples of two-center superdeformed systems which build on an $\alpha+\alpha$ rotor of ${ }^{8} \mathrm{Be}$. Theoretically, molecular orbital (MO), such as the $\pi^{-}$and $\sigma^{+}$orbitals associated with the covalent bonding in atomic molecules, have been successful in understanding the low-lying states of these isotopes [1].

The MO model can describe many kinds of characteristic properties of these isotopes, but they are mainly limited to the analysis on low-lying bound states, and theoretical studies on the highly excited states above the particledecay threshold is still unresearched area. In contrast to the situation of theoretical studies, recent experiments on ${ }^{12} \mathrm{Be}$ revealed the existence of many resonant states [2-4], which strongly decay into ${ }^{6} \mathrm{He}_{\text {g.s. }}+{ }^{6} \mathrm{He}_{\text {g.s. }}$ and $\alpha+{ }^{8} \mathrm{He}_{\text {g.s. }}$. Similar resonances, decaying to $\mathrm{He}$ isotopes, have also been observed in other Be isotopes, such as ${ }^{10} \mathrm{Be}=\alpha+{ }^{6} \mathrm{He}$ and ${ }^{14} \mathrm{Be}={ }^{6} \mathrm{He}+{ }^{8} \mathrm{He}$.

In the present study, we investigate the structural changes appearing in an unbound region of even Be itotopes. In order to investigate the continuum states above the particle decay threshold, the intrinsic structures and their coupling to the scattering states should be treated in a unifie manner. For this purpose, we introduce the generalized twocenter cluster model (GTCM) [5,6]. In this model, the covalent MO configuratio can be smoothly connected to the atomic or ionic one, in which valence neutrons are localized around one of the $\alpha$ cores. Furthermore, it becomes possible to describe both the formation of the covalent MO structures and the reaction process induced by the collision of the ionic state. In ${ }^{12} \mathrm{Be}$ for instance, this model can handle the formation of an atomic structure of ${ }^{6} \mathrm{He}+{ }^{6} \mathrm{He}$,

\footnotetext{
${ }^{a}$ e-mail: itomk@kansai-u.ac.jp
}

ionic structures of $\alpha+{ }^{8} \mathrm{He}$ and ${ }^{5} \mathrm{He}+{ }^{7} \mathrm{He}$, and covalent $\mathrm{MO}$ structures in a consistent manner. In this report, we mainly investigate the intrinsic structure of ${ }^{12} \mathrm{Be}$ and reaction process induced by $\alpha+{ }^{8} \mathrm{He}$ slow collision, and such investigations are extended to even $\mathrm{Be}$ isotope of ${ }^{8,10,12,14,16} \mathrm{Be}$.

\section{Framework}

The detailed explanation of GTCM has already been published in Refs. [5-7], and we briefl show the formulation of GTCM in the following. In GTCM, the total wave function of ${ }^{12} \mathrm{Be}$ is given by the superposition of the basis $\left\{\Phi_{\mathbf{m}}^{J^{\pi} K}(S)\right\}$, where,

$$
\Phi_{\mathbf{m}}^{J^{\pi} K}(S)=\hat{P}_{K}^{J^{\pi}} \mathcal{A}\left\{\psi_{L}(\alpha) \psi_{R}(\alpha) \prod_{j=1}^{4} \varphi_{j}\left(m_{j}\right)\right\}_{S} .
$$

The $\alpha$-cluster $\psi_{n}(\alpha)(n=L, R)$ is expressed by the $(0 \mathrm{~s})^{4}$ configuratio of the harmonic oscillator $(\mathrm{HO})$ centered at the left $(L)$ - or $\operatorname{right}(R)$ - side with the relative distance parameter $S$ [8]. The single-particle wave function for the four valence neutrons localized around one of the $\alpha$ clusters is given by an atomic orbital (AO) $\varphi\left(p_{k}, i, \tau\right)$, and $0 p$-orbitals $p_{k}(k=x, y, z)$ around $i(=L$ or $R)$ with the spin $\tau(=\uparrow$ or $\downarrow)$. Here, $\left\{m_{j}\right\}$ are indices of $\mathrm{AO}\left(p_{k}, i, \tau\right)$ and $\mathbf{m}$ represents a set of AOs for the four neutrons, where $\mathbf{m}=\left(m_{1}, m_{2}, m_{3}, m_{4}\right)$. The intrinsic basis functions with full anti-symmetrization $\mathcal{A}$ are projected to the eigenstate of the total spin $J$, its intrinsic angular projection $K$, and the total parity $\pi$ by the projection operator $\hat{P}_{K}^{J^{\pi}}$. In the present study, we discuss the level structure in the $J^{\pi}=0^{+}$states. Thus, only the case of $J=K=0$ is considered. In the present calculation, we include all possible $\mathrm{AO}$ bases, which have an axial symmetric $K=0$ configuration

The total wave function is finall given by taking the superposition over $S, \mathbf{m}$ as

$$
\Psi^{0_{\nu}^{+}}=\int d S \sum_{\mathbf{m}} C_{\mathbf{m}}^{v}(S) \Phi_{\mathbf{m}}^{0^{+}}(S) \equiv \Psi^{v}
$$


The coefficients for the $0_{\nu}^{+}$state $\left(v\right.$-th $\left.0^{+}\right), C_{\mathbf{m}}^{\nu}(S)$, are determined by solving a coupled channel equation in the Generator Coordinate Method [8].

As for the nucleon-nucleon (NN) interaction, we use the Volkov No.2 and the G3RS for the central and spinorbit parts, respectively. The parameters in the Volkov interactions are $M=0.643, B=H=0.125$, while the strength of G3RS is set to be $+3000 \mathrm{MeV}$ and $-2000 \mathrm{MeV}$ for the repulsive and attractive parts, respectively. The size parameter of $\mathrm{HO}$ is taken to be $b=1.46 \mathrm{fm}$. This parameter set is the same as the set applied in Ref. [5,7], which successfully reproduced the properties of ${ }^{10,12} \mathrm{Be}$.

\section{Results}

\subsection{Results of the intrinsic structures in ${ }^{12} \mathrm{Be}$}

The energy spectra of bound states are obtained by solving the eigenvalue problem with the total wave function in Eq. (2) [5]. In addition, the scattering matrix of the $\alpha+{ }^{8} \mathrm{He}_{\text {g.s. }}$ reaction is calculated by imposing the scattering boundary condition when the system is excited above the threshold energy of this channel $[6,7]$. In the calculation for the $J^{\pi}=0^{+}$state, we identifie the two-bound and four-resonant states below and above the $\alpha+{ }^{8} \mathrm{He}_{\text {g.s. }}$ threshold, respectively.

The ground state has the well developed MO configu ration of $\left(\pi_{3 / 2}^{-}\right)^{2}\left(\sigma_{1 / 2}^{+}\right)^{2}$, and it contains two degree of freedoms such as the $\alpha-\alpha$ relative motion and the single particle motions for the four valence neutrons. By analyzing the properties of the wave functions in the excited states, we fin that all the excited states can be characterized in terms of the excitation degree of freedoms included in the ground states.

We summarized the identifie levels and their intrinsic characters in Fig. 1.

(1) Single particle excitation mode: The $0_{2}^{+}$state has the MO configuration $\left(\pi_{3 / 2}^{-}\right)^{2}\left(\pi_{1 / 2}^{-}\right)^{2}$, while the $0_{5}^{+}$state has a hybrid structure of the $\mathrm{MO}$ and $\mathrm{AO}$ configurations Namely, two of the valence neutrons are localized around individual $\alpha$ as ${ }^{5} \mathrm{He}+{ }^{5} \mathrm{He}$, which is the $\mathrm{AO}$ structure, and the remaining two neutrons occupy the $\sigma^{+}$orbital and rotate around both clusters, which represents the MO character. These states can be characterized as the single particle excitation modes from the ground state.

(2) Cluster excitation mode: The $0_{3}^{+}$and $0_{6}^{+}$states have a large component of the ionic structures of $\alpha+{ }^{8} \mathrm{He}_{\text {g.s. }}$ and ${ }^{5} \mathrm{He}_{\text {g.s. }}+{ }^{7} \mathrm{He}_{\text {g.s. }}$, respectively. These two states are the excitation modes of the $\alpha-\alpha$ relative motion from the $0_{1}^{+}$and $0_{2}^{+}$states, respectively.

(3) Double excitation mode : The $0_{4}^{+}$state has the atomic configuration ${ }^{6} \mathrm{He}_{\text {g.s. }}+{ }^{6} \mathrm{He}_{\text {g.s. }}$. It corresponds to the simultaneous excitation mode of the relative motion of the two $\alpha$-cores and the single particle motions of the excess neutrons.

The result shown in Fig. 1 means that the cluster structures can change from level to level, and various cluster configuration coexist with a small energy interval of about $1 \sim 2 \mathrm{MeV}$ in ${ }^{12} \mathrm{Be}$.

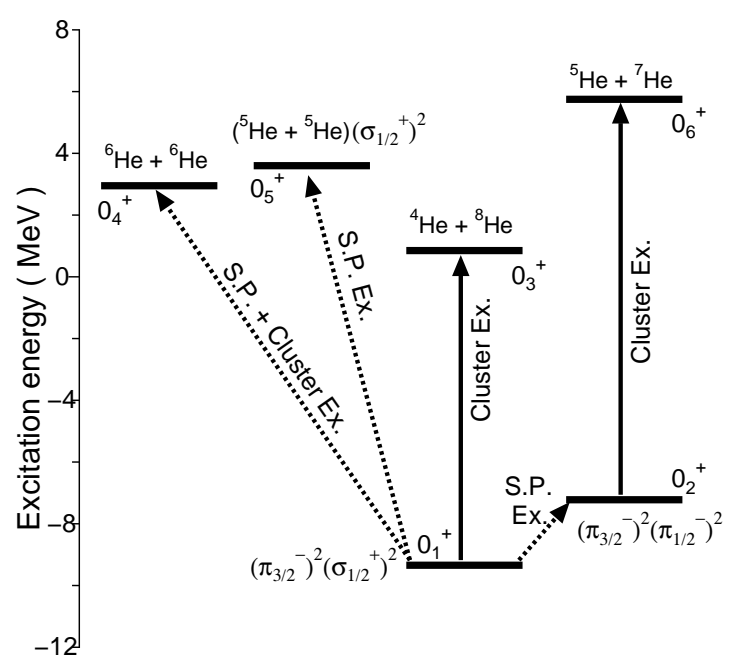

Fig. 1. Energy spectra classifie by the excitation mode $\left(J^{\pi}=0^{+}\right)$. See text for details. The threshold energies of the $\alpha$ emission is taken to be the origins.

\subsection{Monopole strength in ${ }^{12} \mathrm{Be}$}

In ${ }^{12} \mathrm{Be}$, various excited- $0^{+}$states, such as the covalent states $\left(0_{1}^{+}, 0_{2}^{+}\right)$, the ionic states $\left(0_{3}^{+}, 0_{6}^{+}\right)$, the atomic state $\left(0_{4}^{+}\right)$and the covalent-atomic hybrid state $\left(0_{5}^{+}\right)$, appear with small energy spacings $(\sim 1 \sim 2 \mathrm{MeV})$, as shown in Fig. 1. There is a common characteristic property in these $0^{+}$states. All the excited states have a large magnitude of the (isoscalar) monopole transition from the ground $(v=1)$ state [10], which is define by

$$
\begin{aligned}
& M(I S) \\
& \quad=<\Psi^{v}\left|\sum_{i=1}^{12} r_{i}^{2}\right| \Psi^{1}>\equiv<0_{v}^{+}\left|\sum_{i=1}^{12} r_{i}^{2}\right| 0_{1}^{+}>.
\end{aligned}
$$

Here, $r_{i}$ denotes the radial coordinate for the $i$-th nucleon in ${ }^{12} \mathrm{Be}$. In general neutron-excess systems, an isovector part of monopole matrix elements does not vanish and contributes to the total strength of a monopole transition. However, the isovector excitation becomes zero if the target nucleus is an isospin-saturated system, such as the $\alpha$ particle. In recent experiments, nuclear reactions with an $\alpha$ target are often employed to probe excited states of neutron excess systems [4]. In this study, therefore, we consider only the isoscalar part of the monopole matrix element.

In order to discuss the magnitude of the monopole strength clearly, we show the ratio of the calculated strength to the respective single particle strength, $\left|M(I S) / M^{s \cdot p} \cdot\right|$, in Table 1 . The single particle strength is define by the simple HO wave function in the $p$-shell [9] as:

$$
M^{s . p .}=<1 p, b\left|r^{2}\right| 0 p, b>=2 b^{2} \sqrt{\frac{5}{8}} .
$$


Here, $b$ is a size parameter of $\mathrm{HO}(b=1.46 \mathrm{fm})$, and $0 p$ and $1 p$ denote the radial wave function of $\mathrm{HO}$ with the zero and one radial node, respectively.

\begin{tabular}{|c|c|}
\hline State & Ratio \\
\hline $0_{2}^{+}$ & 2.59 \\
\hline $0_{3}^{+}$ & 3.53 \\
\hline $0_{4}^{+}$ & 0.92 \\
\hline $0_{5}^{+}$ & 1.48 \\
\hline $0_{6}^{+}$ & 1.76 \\
\hline
\end{tabular}

Table 1. The ratio of the total monopole strength and the single particle strength in the $0 p$-shell.

All the strength is comparable to or a few times larger than the single particle strength, and these values are almost the same magnitude as the transition to the $0_{2}^{+}$state at $E_{x}=7.65 \mathrm{MeV}$ in ${ }^{12} \mathrm{C}$, having a $3 \alpha$ cluster structure. The ratio of the monopole strength of $0_{1}^{+} \rightarrow 0_{2}^{+}$is about 4.6 [11]. This result is consistent to the analysis of the monopole strength in Ref. [9], where the enhancement of the lowlying monopole strength is discussed by cluster formations. It should be noted that all the monopole strength, corresponding to the various cluster structures, appears at $E_{x} \leq$ $20 \mathrm{MeV}$ in the present system. In marked contrast to this result, in a naive mean fiel picture, a $2 \hbar \omega(\sim 35 \mathrm{MeV})$ jump is needed for monopole excitations. This means that it is quite difficult to explain the monopole strength shown in Table 1 by mean fiel models and hence, the present result is considered to be abnormal in a naive single particle picture.

In a system with a considerable neutron excess, there are almost degenerate monopole states at much lower energies than expected in a naive shell-model picture, and the transition strengths to these states are comparable with the single-particle strength.

Among the various $0^{+}$states, strong enhancement occurs for the transition to the $0_{3}^{+}$state $\left(\alpha+{ }^{8} \mathrm{He}_{\text {g.s. }}\right)$, which is a direct cluster's excitation mode from the ground state. An enhancement can also be seen for the $0_{2}^{+}$state with the two neutrons' excited configuration $\left(\pi_{3 / 2}^{-}\right)^{2}\left(\pi_{1 / 2}^{-}\right)^{2}$. This enhancement is due to the mixing of $\left(\pi_{3 / 2}^{-}\right)^{2}\left(\sigma_{1 / 2}^{+}\right)^{2}$ and $\left(\pi_{3 / 2}^{-}\right)^{2}\left(\pi_{1 / 2}^{-}\right)^{2}$ as pointed out in Ref. [12]. However, the strength for $0_{3}^{+}$is about two times the magnitude of the strength for $0_{2}^{+}$, and the transition to the cluster excited state, $0_{3}^{+}$, is strongest in all the monopole strength.

\subsection{Results of even $\mathrm{Be}$ isotopes}

We apply the GTCM calculations to even Be isotopes, ${ }^{8 \sim 16} \mathrm{Be}$. The energy spectra of ${ }^{10} \mathrm{Be}$ with $J^{\pi}=0^{+}$is shown in Fig. 2 . The total reaction probability is define by $1-\mathfrak{R} S_{e l}$, in which $S_{e l}$ denotes the scattering matrices of the elastic scattering of $\alpha+{ }^{6} \mathrm{He}$, and it is shown by the dotted curve at the right part in Fig. 2.

Below the $\alpha$ decay threshold shown by the thin solid line, two bound states appear. The intrinsic structure of the

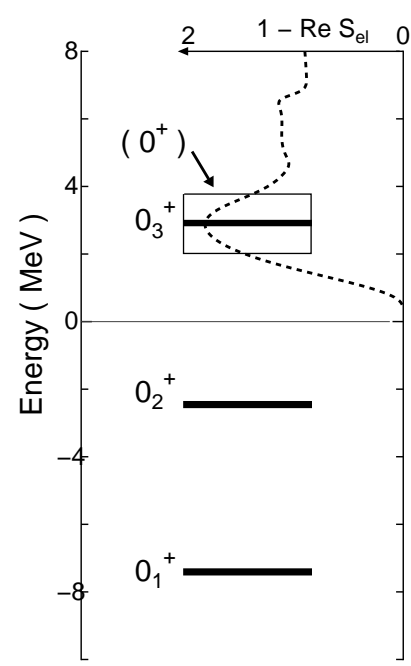

Fig. 2. (a) Energy spectra of ${ }^{10} \mathrm{Be}\left(J^{\pi}=0^{+}\right)$. The threshold energies of the $\alpha+{ }^{6} \mathrm{He}_{\text {g.s. }}$ channel is taken to be the origins. The dotted curve at the right part represents the reaction probability for the central collision of $\alpha+{ }^{6} \mathrm{He}_{\text {g.s. }}$. See text for details.

$0_{1}^{+}$and $0_{2}^{+}$states are nicely described by the $\mathrm{MO}$ config uration of $\left(\pi_{3 / 2}^{-}\right)^{2}$ and $\left(\sigma_{1 / 2}^{+}\right)^{2}$, respectively. Since the $\sigma_{1 / 2}^{+}$ orbit has an enlarged distribution along the $\alpha-\alpha$ axis, the $\alpha$ $\alpha$ clustering is well developed in the $0_{2}^{+}$state. Above the $\alpha$ decay threshold, the ionic states are realized as continuum resonant states. In the total reaction probability, a prominent peak appears around $E \sim 3 \mathrm{MeV}$. This enhancement of the reaction probability is due to the formation of the resonance with the ionic structure of $\alpha+{ }^{6} \mathrm{He}\left(2_{1}^{+}\right)$, which is shown by the solid energy level of $0_{3}^{+}$in Fig. 2 . In addition, at the same energy region, there is a broad continuum strength, which has the ionic structure of $\alpha+{ }^{6} \mathrm{He}_{\text {g.s. }}$. This continuum state is shown by a transparent square and $0^{+}$in parenthesis.

Two $0^{+}$states embedded in continuum correspond to the cluster's relative excitation mode from the bound states: The $0_{3}^{+}$state is cluster's excitation from the ground $0_{1}^{+}$state, while the continuum $\left(0^{+}\right)$can be considered as the similar excitation mode from the $0_{2}^{+}$state. These states are just analog states to the ionic state, $\alpha+{ }^{8} \mathrm{He}_{\text {g.s. }}\left(0_{3}^{+}\right)$and ${ }^{5} \mathrm{He}_{\text {g.s. }}+{ }^{7} \mathrm{He}_{\text {g.s. }}\left(0_{6}^{+}\right)$, in ${ }^{12} \mathrm{Be}$.

The $J^{\pi}=0^{+}$energy spectra for ${ }^{14} \mathrm{Be}$ is shown in Fig. 3 . In this figure we can clearly confir the similar level structure to ${ }^{10} \mathrm{Be}$. In the bound region, two $0^{+}$states appears, while two resonant states are embedded in continuum energy. The ground $0_{1}^{+}$state has a spatially compact structure, which can be nicely understood by the shell model picture. However, the $0_{2}^{+}$state has a well developed $\alpha-\alpha$ cluster structure. Furthermore, two resonant states, which have the ionic structures, are identifie by solving the ${ }^{6} \mathrm{He}+{ }^{8} \mathrm{He}$ collision. These resonant states correspond to the cluster's excitation modes from the bound states: The $0_{3}^{+}$state, which has the ionic structure of ${ }^{6} \mathrm{He}_{\text {g.s. }}+{ }^{8} \mathrm{He}_{\text {g.s. }}$, is the cluster's excitation mode from the $0_{2}^{+}$state, while the $0_{4}^{+}$state has the ${ }^{6} \mathrm{He}\left(2_{1}^{+}\right)+{ }^{8} \mathrm{He}_{\text {g.s. }}$ structure and is the excitation mode from the ground $0_{1}^{+}$state. 


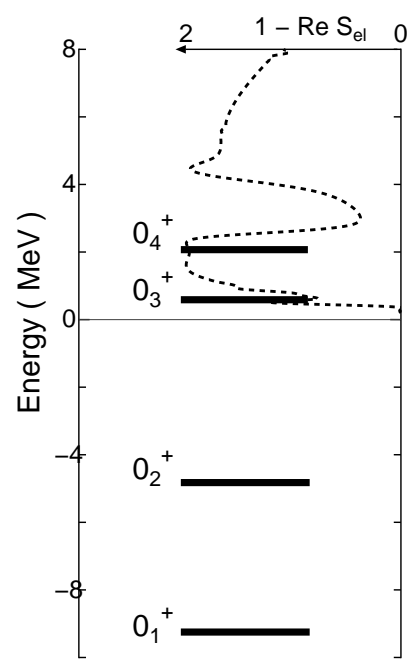

Fig. 3. The same figur as Fig. 2 but for ${ }^{14} \mathrm{Be}$. See test for details.

In ${ }^{10} \mathrm{Be}$, one of two $0^{+}$states above the $\alpha$ threshold becomes a broad continuum state, but, in ${ }^{14} \mathrm{Be}$, both unbound $0^{+}$states becomes the resonant states. This is because of the increase of excess neutrons. Due to the increase of excess neutrons, the attraction between ${ }^{6} \mathrm{He}$ and ${ }^{8} \mathrm{He}$ in ${ }^{14} \mathrm{Be}$ is larger than that between $\alpha$ and ${ }^{6} \mathrm{He}$ in ${ }^{10} \mathrm{Be}$. Thus, unbound $0^{+}$states are stabilized as the resonant states in ${ }^{14} \mathrm{Be}$.

We also analyzed the level structure of ${ }^{16} \mathrm{Be}$ by applying the GTCM. There is a possibility of the resonance formation of a nuclear dimer such as ${ }^{8} \mathrm{He}+{ }^{8} \mathrm{He}$ although ${ }^{16} \mathrm{Be}$ itself is an unstable nucleus with respect to two neutrons emission. The ratio of $N / A$ in the ${ }^{8} \mathrm{He}$ nucleus is 0.75 , which is the highest value in bound nuclear systems. Thus, the ${ }^{8} \mathrm{He}+{ }^{8} \mathrm{He}$ atomic state corresponds to the nuclear dimers with extremely neutron excess, and it is very interesting to investigate the possibility of its formation.

We have found that the ground state in ${ }^{16} \mathrm{Be}$ appears below the ${ }^{8} \mathrm{He}_{\text {g.s. }}+{ }^{8} \mathrm{He}_{\text {g.s. }}$ threshold by about $4 \mathrm{MeV}$. This ground state corresponds to the unbound states above the ${ }^{14} \mathrm{Be}_{\text {g.s. }}+2 \mathrm{n}$ threshold by about $3 \mathrm{MeV}$. It is well known that, in ${ }^{8} \mathrm{Be}$, the sharp resonance with the $\alpha+\alpha$ structure exists just above the two $\alpha$ threshold. The ground state in ${ }^{16} \mathrm{Be}$, which is predicted by the GTCM calculation, can be considered as an analog state of $\alpha+\alpha$ in ${ }^{8} \mathrm{Be}$. Due to the effect of excess neutrons, the unbound resonance of $\alpha+\alpha$ becomes a "bound state" below the ${ }^{8} \mathrm{He}_{\text {g.s. }}+{ }^{8} \mathrm{He}_{\text {g.s. }}$. Above the threshold, we have confirme some resonance like behaviors in the scattering matrix of ${ }^{8} \mathrm{He}+{ }^{8} \mathrm{He}$. The analysis of the wave function in ${ }^{16} \mathrm{Be}$ is now under way.

\section{Summary}

In summary, we have studied the exotic structures of ${ }^{12} \mathrm{Be}$ as an unbound system by applying GTCM. Due to the excitation of the cluster and single particle degrees of freedom, various structures appear as a variation of the excitation energy. Furthermore, above the particle decay threshold, we found that excited states coexist within the much small energy. Individual excited states can be characterized in terms of the excitation mode of the cluster's or excess neutron's degrees of freedom. All the energy levels, which appear with close energy spacings, have the monopole strength comparable to or larger than the single particle strength. This means that, in light neutron excess systems, strong monopole strength can be observed in the lower energy region, and this feature is completely consistent with recent observation of monopole breakups in ${ }^{12} \mathrm{Be}$ [4].

Systematic calculation is applied on even Be isotopes. We have found that, in ${ }^{10,14} \mathrm{Be}$, the ionic structures, which are embedded in continuum, are realized as the cluster's excitation modes from the bound states. In ${ }^{16} \mathrm{Be}$, an energy level, which can be considered as the $\alpha+\alpha$ analog state, is obtained in ${ }^{16} \mathrm{Be}$. From the systematic studies, we obtained a wide variety of cluster structures in even $\mathrm{Be}$ isotopes. Such various structures can also be expected in other neutron excess systems, such as $\mathrm{O}$ isotopes $\left(\alpha+{ }^{12} \mathrm{C}+\mathrm{XN}\right)$ and $\mathrm{Ne}$ isotopes $\left(\alpha+{ }^{16} \mathrm{O}+\mathrm{X} N\right)$. Systematic studies on $\mathrm{O}$ and $\mathrm{Ne}$ isotopes are now proceeding.

\section{References}

1. N. Itagaki and S. Okabe, Phys. Rev. C 61, 044306 (2000). N. Itagaki, S. Okabe and K. Ikeda, Phys. Rev. C 62, 034301 (2000), and references therein.

2. A. A. Korsheninnikov et al., Phys. Lett. B 343, 53 (1995).

3. M. Freer et al., Phys. Rev. C 63, 034301 (2001).

4. A. Saito et al., Suppl. Prog. Theor. Phys., 146, 615 (2003). A. Saito et al., Mod. Phys. Lett. A 25, 21 (2010).

5. M. Ito, K. Kato and K. Ikeda, Phys. Lett. B588, 43 (2004); Makoto Ito, Phys. Lett. B636, 293 (2006).

6. M. Ito, N. Itagaki, H. Sakurai, and K. Ikeda, Phys. Rev. Lett. 100, 182502 (2008);M. Ito and N. Itagaki, Phys. Rev. C 78, 011602(R) (2008); ibid, Phys. Rev. Focus Vol. 22, Story 4 (2008).

7. Makoto Ito, Phys. Lett. B 636, 293 (2006); M. Ito and K. Yabana, Prog. Theor. Phys. 113, 1047 (2005)

8. H. Horiuchi et al., Suppl. Prog. Theor. Phys. 62, 1 (1977).

9. T. Yamada, Y. Funaki, H. Horiuchi, K. Ikeda, Y. Funaki, and A. Thosaki, Prog. Theor. Phys. 120, 1139 (2008).

10. Makoto Ito, Phys. Rev. C 83, 044319 (2011).

11. M. Kamimura, Nucl. Phys. A351, 456 (1981).

12. Shimoura et al., Phys. Lett. B 654, 87 (2007). 